\title{
Time-Fractional Optimal Control of Initial Value Problems on Time Scales*
}

\author{
Gaber M. Bahaa and Delfim F. M. Torres
}

\begin{abstract}
We investigate Optimal Control Problems (OCP) for fractional systems involving fractional-time derivatives on time scales. The fractional-time derivatives and integrals are considered, on time scales, in the Riemann-Liouville sense. By using the Banach fixed point theorem, sufficient conditions for existence and uniqueness of solution to initial value problems described by fractional order differential equations on time scales are known. Here we consider a fractional OCP with a performance index given as a delta-integral function of both state and control variables, with time evolving on an arbitrarily given time scale. Interpreting the Euler-Lagrange first order optimality condition with an adjoint problem, defined by means of right Riemann-Liouville fractional delta derivatives, we obtain an optimality system for the considered fractional OCP. For that, we first prove new fractional integration by parts formulas on time scales.
\end{abstract}

Keywords: fractional derivatives and integrals on time scales, initial value problems, optimal control.

2010 Mathematics Subject Classification: 26A33, 34N05, 49K99.

Gaber M. Bahaa

Department of Mathematics and Computer Science, Faculty of Science, Beni-Suef University, BeniSuef, Egypt; and Department of Mathematics, Faculty of Science, Taibah University, Al-Madinah Al-Munawarah, Saudi Arabia; e-mail: Bahaa\$_\{-\}\$gm@yahoo.com

Delfim F. M. Torres

Center for Research and Development in Mathematics and Applications (CIDMA), Department of Mathematics, University of Aveiro, 3810-193 Aveiro, Portugal; e-mail: delfim@ua.pt

* This is a preprint of a paper accepted for publication as a book chapter with Springer International Publishing AG. Submitted 23/Jan/2019; revised 27-March-2019; accepted 12-April-2019. 


\section{Introduction}

Let $\mathbb{T}$ be a time scale, that is, a nonempty closed subset of $\mathbb{R}$. We consider the following initial value problem:

$$
\begin{gathered}
{ }_{t_{0}}^{\mathbb{T}} D_{t}^{\alpha} y(t)=f(t, y(t)), \quad t \in\left[t_{0}, t_{0}+a\right]=\mathcal{J} \subseteq \mathbb{T}, \quad 0<\alpha<1, \\
{ }_{t_{0}}^{\mathbb{T}} I_{t}^{1-\alpha} y\left(t_{0}\right)=0,
\end{gathered}
$$

where ${ }_{t_{0}}^{\mathbb{T}} D_{t}^{\alpha}$ is the (left) Riemann-Liouville fractional derivative operator or order $\alpha$ defined on $\mathbb{T}$ and ${ }_{t_{0}}^{\mathbb{T}} I_{t}^{1-\alpha}$ is the (left) Riemann-Liouville fractional integral operator or order $1-\alpha$ defined on $\mathbb{T}$, as introduced in [24] (see also [45, 46]), and function $f: \mathcal{J} \times \mathbb{R} \rightarrow \mathbb{R}$ is a right-dense continuous function. Necessary and sufficient conditions for the existence and uniqueness of solution to problem (1) are already discussed in [24]. Here, our goal is to prove optimality conditions for such systems.

Fractional Calculus (FC) is a generalization of classical calculus. It has been reported in the literature that systems described using fractional derivatives give a more realistic behavior. There exists many definitions of a fractional derivative. Commonly used fractional derivatives are the classical Riemann-Liouville and Caputo derivatives on continuous time scales. Fractional derivatives and integrals of Riemann-Liouville and Caputo types have a vast number of applications, across many fields of science and engineering. For example, they can be used to model controllability, viscoelastic flows, chaotic systems, Stokes problems, thermo-elasticity, several vibration and diffusion processes, bioengineering problems, and many other complex phenomena: see, e.g., [6, 11] and references therein.

Fractional optimal control problems on a continuous time scale have attracted several authors in the last two decades, and many techniques have been developed for solving such problems, involving classical fractional derivatives. Agrawal [6, 7] presented a general formulation and proposed a numerical method to solve such problems. In those papers, the fractional derivative was defined in the Riemann-Liouville sense and the formulation was obtained by means of a fractional variational principle and the Lagrange multiplier technique. Using new techniques, Frederico and Torres [32, 33] obtained Noether-like theorems for fractional optimal control problems in both Riemann-Liouville and Caputo senses. In [39, 40], Mophou and N'Guérékata studied the fractional optimal control of diffusion equations involving the classical Riemann-Liouville derivatives. In [43], Ozdemir investigated the fractional optimal control problem of a distributed system in cylindrical coordinates whose dynamics are defined in the classical Riemann-Liouville sense. For the state of the art and many generalizations, see the recent books [9, 37].

The theory of fractional differential equations, specifically the question of existence and uniqueness of solutions, is a research topic of great importance [1, 12, 34]. Another important area of study is dynamic equations on time scales, which goes back to 1988 and the work of Aulbach and Hilger, and has been used with success to unify differential and difference equations [5, 10, 26]. Starting with a linear dynamic equation, Bastos et al. have introduced the notion of fractional-order deriva- 
tive on time scales, involving time-scale analogues of Riemann-Liouville operators [15, 16, 17]. Another approach originate from the inverse Laplace transform on time scales [18]. After such pioneer work, the study of fractional calculus on time scales developed in a popular research subject: see [20, 22, 23, 25, 42] and the more recent references [2, 19, 21, 38, 41].

To the best of our knowledge, the study of fractional optimal control problems for dynamical systems on time scales is under-developed, at least when compared to the continuous and discrete cases [31, 36]. Motivated by this fact, in this paper an Optimal Control Problem (OCP) for fractional initial value systems involving fractional-time derivatives on time scales is considered. The fractional-time derivative and integral are considered in the Riemann-Liouville sense on time scales, as introduced in [24]. We prove necessary optimality conditions for such OCPs. The performance index of the Fractional Optimal Control Problem (FOCP) is considered as a non-autonomous delta integral of a function depending on state and control variables, and where the dynamic control system is expressed by a delta-differential system. Interpreting the Euler-Lagrange first order optimality condition with an adjoint problem, defined by means of the time-scale right fractional derivative in the sense of Riemann-Liouville, we obtain an optimality system for the FOCP on time scales.

\section{Preliminaries}

In this section, we collect notations, definitions, and results, which are needed in the sequel. We use $C(\mathcal{J}, \mathbb{R})$ for the Banach space of continuous functions $y$ with the norm $\|y\|_{\infty}=\sup \{|y(t)|: t \in \mathcal{J}\}$, where $\mathcal{J}$ is a time-scale interval.

\subsection{Time-scale essentials}

A time scale $\mathbb{T}$ is an arbitrary nonempty closed subset of $\mathbb{R}$. The reader interested on the calculus on time scales is referred to the books [26, 27]. For a survey, see [5]. Any time scale $\mathbb{T}$ is a complete metric space with the distance $d(t, s)=|t-s|, t, s \in \mathbb{T}$. Consequently, according to the well-known theory of general metric spaces, we have for $\mathbb{T}$ the fundamental concepts such as open balls (intervals), neighborhoods of points, open sets, closed sets, compact sets, etc. In particular, for a given number $\delta>0$, the $\delta$-neighborhood $\mathrm{U}_{\delta}(t)$ of a given point $t \in \mathbb{T}$ is the set of all points $s \in \mathbb{T}$ such that $d(t, s)<\delta$. We also have, for functions $f: \mathbb{T} \rightarrow \mathbb{R}$, the concepts of limit, continuity, and the properties of continuous functions on a general complete metric space. Roughly speaking, the calculus on time scales begins by introducing and investigating the concept of derivative for functions $f: \mathbb{T} \rightarrow \mathbb{R}$. In the definition of derivative, an important role is played by the so-called jump operators. 
Definition 1 (See [27]). Let $\mathbb{T}$ be a time scale. For $t \in \mathbb{T}$, we define the forward jump operator $\sigma: \mathbb{T} \rightarrow \mathbb{T}$ by $\sigma(t):=\inf \{s \in \mathbb{T}: s>t\}$, and the backward jump operator $\rho: \mathbb{T} \rightarrow \mathbb{T}$ by $\rho(t):=\sup \{s \in \mathbb{T}: s<t\}$.

Remark 1 In Definition 1 we put inf $\emptyset=\sup \mathbb{T}$ (i.e., $\sigma(M)=M$ if $\mathbb{T}$ has a maximum $M)$ and $\sup \emptyset=\inf \mathbb{T}$ (i.e., $\rho(m)=m$ if $\mathbb{T}$ has a minimum $m$ ).

If $\sigma(t)>t$, then we say that $t$ is right-scattered; if $\rho(t)<t$, then $t$ is said to be left-scattered. Points that are simultaneously right-scattered and left-scattered are called isolated. If $t<\sup \mathbb{T}$ and $\sigma(t)=t$, then $t$ is called right-dense; if $t>\inf \mathbb{T}$ and $\rho(t)=t$, then $t$ is called left-dense. The graininess function $\mu: \mathbb{T} \rightarrow[0, \infty)$ is defined by $\mu(t):=\sigma(t)-t$. The derivative makes use of the set $\mathbb{T}^{\kappa}$, which is obtained from the time scale $\mathbb{T}$ as follows: if $\mathbb{T}$ has a left-scattered maximum $M$, then $\mathbb{T}^{K}:=\mathbb{T} \backslash\{M\} ;$ otherwise, $\mathbb{T}^{\kappa}:=\mathbb{T}$.

Definition 2 (Delta derivative [4]) Assume $f: \mathbb{T} \rightarrow \mathbb{R}$ and let $t \in \mathbb{T}^{K}$. We define

$$
f^{\Delta}(t):=\lim _{s \rightarrow t} \frac{f(\sigma(s))-f(t)}{\sigma(s)-t}, \quad t \neq \sigma(s),
$$

provided the limit exists. We call $f^{\Delta}(t)$ the delta derivative (or Hilger derivative) of $f$ at $t$. Moreover, we say that $f$ is delta differentiable on $\mathbb{T}^{\kappa}$ provided $f^{\Delta}(t)$ exists for all $t \in \mathbb{T}^{\kappa}$. The function $f^{\Delta}: \mathbb{T}^{K} \rightarrow \mathbb{R}$ is then called the (delta) derivative of $f$ on $\mathbb{T}^{\kappa}$.

Definition 3 (See [27]). A function $f: \mathbb{T} \rightarrow \mathbb{R}$ is called rd-continuous provided it is continuous at right-dense points in $\mathbb{T}$ and its left-sided limits exist (finite) at leftdense points in $\mathbb{T}$. The set of rd-continuous functions $f: \mathbb{T} \rightarrow \mathbb{R}$ is denoted by $C_{r d}$. Similarly, a function $f: \mathbb{T} \rightarrow \mathbb{R}$ is called ld-continuous provided it is continuous at left-dense points in $\mathbb{T}$ and its right-sided limits exist (finite) at right-dense points in $\mathbb{T}$. The set of ld-continuous functions $f: \mathbb{T} \rightarrow \mathbb{R}$ is denoted by $C_{l d}$.

Definition 4 (See [27]). Let $[a, b]$ denote a closed bounded interval in $\mathbb{T}$. A function $F:[a, b] \rightarrow \mathbb{R}$ is called a delta antiderivative of function $f:[a, b) \rightarrow \mathbb{R}$ provided $F$ is continuous on $[a, b]$, delta differentiable on $[a, b)$, and $F^{\Delta}(t)=f(t)$ for all $t \in[a, b)$. Then, we define the $\Delta$-integral of $f$ from $a$ to $b$ by

$$
\int_{a}^{b} f(t) \Delta t:=F(b)-F(a) .
$$

Proposition 1 (See [8]) Suppose $\mathbb{T}$ is a time scale and $f$ is an increasing continuous function on the time-scale interval $[a, b]$. If $F$ is the extension of $f$ to the real interval $[a, b]$ given by

$$
F(s):= \begin{cases}f(s) & \text { if } s \in \mathbb{T} \\ f(t) & \text { if } s \in(t, \sigma(t)) \notin \mathbb{T}\end{cases}
$$

then

$$
\int_{a}^{b} f(t) \Delta t \leq \int_{a}^{b} F(t) d t
$$




\subsection{Fractional derivative and integral on time scales}

We adopt a recent notion of fractional derivative on time scales introduced in [24], which is based on the notion of fractional integral on time scales $\mathbb{T}$. This is in contrast with [22, 23, 25], where first a notion of fractional differentiation on time scales is introduced and only after that, with the help of such concept, the fraction integral is defined. The classical gamma and beta functions are used.

Definition 5 (Gamma function) For complex numbers with a positive real part, the gamma function $\Gamma(t)$ is defined by the following convergent improper integral:

$$
\Gamma(t):=\int_{0}^{\infty} x^{t-1} e^{-x} d x
$$

Definition 6 (Beta function) The beta function, also called the Euler integral of first kind, is the special function $\mathrm{B}(x, y)$ defined by

$$
\mathrm{B}(x, y):=\int_{0}^{1} t^{x-1}(1-t)^{y-1} d t, \quad x>0, \quad y>0 .
$$

Remark 2 The gamma function satisfies the following property: $\Gamma(t+1)=t \Gamma(t)$. The beta function can be expressed through the gamma function by

$$
\mathrm{B}(x, y)=\frac{\Gamma(x) \Gamma(y)}{\Gamma(x+y)} .
$$

Definition 7 (Fractional integral on time scales [24]) Suppose $\mathbb{T}$ is a time scale, $[a, b]$ is an interval of $\mathbb{T}$, and $h$ is an integrable function on $[a, b]$. Let $0<\alpha<1$. Then the left fractional integral of order $\alpha$ of $h$ is defined by

$$
{ }_{a}^{\mathbb{T}} I_{t}^{\alpha} h(t):=\int_{a}^{t} \frac{(t-s)^{\alpha-1}}{\Gamma(\alpha)} h(s) \Delta s
$$

The right fractional integral of order $\alpha$ of $h$ is defined by

$$
{ }_{t}^{\mathbb{T}} I_{b}^{\alpha} h(t):=\int_{t}^{b} \frac{(s-t)^{\alpha-1}}{\Gamma(\alpha)} h(s) \Delta s,
$$

where $\Gamma$ is the gamma function.

Definition 8 (Riemann-Liouville fractional derivative on time scales [24]) Let $\mathbb{T}$ be a time scale, $t \in \mathbb{T}, 0<\alpha<1$, and $h: \mathbb{T} \rightarrow \mathbb{R}$. The left Riemann-Liouville fractional derivative of order $\alpha$ of $h$ is defined by

$$
{ }_{a}^{\mathbb{T}} D_{t}^{\alpha} h(t):=\left({ }_{a}^{\mathbb{T}} I_{t}^{1-\alpha} h(t)\right)^{\Delta}=\frac{1}{\Gamma(1-\alpha)}\left(\int_{a}^{t}(t-s)^{-\alpha} h(s) \Delta s\right)^{\Delta} .
$$

The right Riemann-Liouville fractional derivative of order $\alpha$ of $h$ is defined by 


$$
{ }_{t}^{\mathbb{T}} D_{b}^{\alpha} h(t):=-\left({ }_{t}^{\mathbb{T}} I_{b}^{1-\alpha} h(t)\right)^{\Delta}=\frac{-1}{\Gamma(1-\alpha)}\left(\int_{t}^{b}(s-t)^{-\alpha} h(s) \Delta s\right)^{\Delta} .
$$

Definition 9 (Caputo fractional derivative on time scales [8]) Let $\mathbb{T}$ be a time scale, $t \in \mathbb{T}, 0<\alpha<1$, and $h: \mathbb{T} \rightarrow \mathbb{R}$. The left Caputo fractional derivative of order $\alpha$ of $h$ is defined by

$$
{ }_{a}^{\mathbb{T} C} D_{t}^{\alpha} h(t):={ }_{a}^{\mathbb{T}} I_{t}^{1-\alpha}\left(h^{\Delta}(t)\right)=\frac{1}{\Gamma(1-\alpha)} \int_{a}^{t}(t-s)^{-\alpha} h^{\Delta}(s) \Delta s .
$$

The right Caputo fractional derivative of order $\alpha$ of $h$ is defined by

$$
{ }_{t}^{\mathbb{T} C} D_{b}^{\alpha} h(t):=-{ }_{t}^{\mathbb{T}} I_{b}^{1-\alpha}\left(h^{\Delta}(t)\right)=\frac{-1}{\Gamma(1-\alpha)} \int_{t}^{b}(s-t)^{-\alpha} h^{\Delta}(s) \Delta s .
$$

The relation between the left/right RLFD and the left/right CFD is as follows:

$$
\begin{aligned}
& { }_{a}^{\mathbb{T} C} D_{t}^{\alpha} x(t)={ }_{a}^{\mathbb{T}} D_{t}^{\alpha} x(t)-\sum_{k=0}^{n-1} \frac{x^{(k)}(a)}{\Gamma(k-\alpha+1)}(t-a)^{(k-\alpha)}, \\
& { }_{t}^{\mathbb{T} C} D_{b}^{\alpha} x(t)={ }_{t}^{\mathbb{T}} D_{b}^{\alpha} x(t)-\sum_{k=0}^{n-1} \frac{x^{(k)}(b)}{\Gamma(k-\alpha+1)}(b-t)^{(k-\alpha)} .
\end{aligned}
$$

If $x$ and $x^{(i)}, i=1, \ldots, n-1$, vanish at $t=a$, then ${ }_{a}^{\mathbb{T}} D_{t}^{\beta} x(t)={ }_{a}^{\mathbb{T} C} D_{t}^{\beta} x(t)$, and if they vanish at $t=b$, then ${ }_{t}^{\mathbb{T}} D_{b}^{\beta} x(t)={ }_{t}^{\mathbb{T} C} D_{b}^{\beta} x(t)$. Furthermore, ${ }_{a}^{\mathbb{T} C} D_{t}^{\alpha} c=0$, where $c$ is a constant, and

$$
{ }_{a}^{\mathbb{T} C} D_{t}^{\alpha} t^{n}=\left\{\begin{array}{l}
0, \text { for } n \in N_{0} \text { and } n<[\alpha], \\
\frac{\Gamma(n+1)}{\Gamma(n+1-\alpha)} t^{n-\alpha}, \text { for } n \in \mathbb{N}_{0} \text { and } n \geq[\beta],
\end{array}\right.
$$

where $\mathbb{N}_{0}=\{0,1,2, \ldots\}$.

Remark 3 If $\mathbb{T}=\mathbb{R}$, then Definition 8 gives the classical left and right RiemannLiouville fractional derivatives [44]. Similar comment for Definition9 For different extensions of the fractional derivative to time scales using the Caputo approach, see [18]. For local approaches to fractional calculus on time scales, we refer the reader to [22, 23, 25]. Here we restrict ourselves to the delta approach to time scales. Analogous definitions are, however, trivially obtained for the nabla approach to time scales by using the duality theory of [3, 30].

\subsection{Properties of the time-scale fractional operators}

We recall some fundamental properties of the fractional operators on time scales. 
Proposition 2 (See Proposition 15 of [24]). Let $\mathbb{T}$ be a time scale with derivative $\Delta$, and $0<\alpha<1$. Then, ${ }_{a}^{\mathbb{T}} D_{t}^{\alpha}=\Delta \circ{ }_{a}^{\mathbb{T}} I_{t}^{1-\alpha}$.

Proposition 3 (See Proposition 16 of [24]). For any function $h$ integrable on $[a, b]$, the Riemann-Liouville $\Delta$-fractional integral satisfies ${ }_{a}^{\mathbb{T}} I_{t}^{\alpha} \circ{ }_{a}^{\mathbb{T}} I_{t}^{\beta}={ }_{a}^{\mathbb{T}} I_{t}^{\alpha+\beta}$ for $\alpha>0$ and $\beta>0$.

Proposition 4 (See Proposition 17 of [24]). For any function $h$ integrable on $[a, b]$ one has ${ }_{a}^{\mathbb{T}} D_{t}^{\alpha} \circ{ }_{a}^{\mathbb{T}} I_{t}^{\alpha} h=h$.

Corollary 1 (See Corollary 18 of [24]). For $0<\alpha<1$, we have ${ }_{a}^{\mathbb{T}} D_{t}^{\alpha} \circ{ }_{a}^{\mathbb{T}} D_{t}^{-\alpha}=I d$ and ${ }_{a}^{\mathbb{T}} I_{t}^{-\alpha} \circ{ }_{a}^{\mathbb{T}} I_{t}^{\alpha}=I d$, where Id denotes the identity operator.

Definition 10 (See [24]) For $\alpha>0$, we denote by ${ }_{a}^{\mathbb{T}} I_{t}^{\alpha}([a, b])$ the space of functions that can be represented by the Riemann-Liouville $\Delta$ integral of order $\alpha$ of some $C([a, b])$-function.

Theorem 1 (See Theorem 20 of [24]). Let $f \in C([a, b])$ and $\alpha>0$. Function $f \in{ }_{a}^{\mathbb{T}} I_{t}^{\alpha}([a, b])$ if and only if ${ }_{a}^{\mathbb{T}} I_{t}^{1-\alpha} f \in C^{1}([a, b])$ and $\left.\left({ }_{a}^{\mathbb{T}} I_{t}^{1-\alpha} f(t)\right)\right|_{t=a}=0$.

Theorem 2 (See Theorem 21 of [24]) Let $\alpha>0$ and $f \in C([a, b])$ satisfy the conditions in Theorem 1 Then, $\left({ }_{a}^{\mathbb{T}} I_{t}^{\alpha} \circ{ }_{a}^{\mathbb{T}} D_{t}^{\alpha}\right)(f)=f$.

\subsection{Existence of solutions to fractional IVPs on time scales}

Let $\mathbb{T}$ be a time scale and $\mathcal{J}=\left[t_{0}, t_{0}+a\right] \subset \mathbb{T}$. Consider the fractional order initial value problem (1) defined on $\mathbb{T}$. Then the function $y \in C(\mathcal{J}, \mathbb{R})$ is a solution of problem (1) if ${ }_{t_{0}}^{\mathbb{T}} D_{t}^{\alpha} y(t)=f(t, y)$ on $\mathcal{J}$ and ${ }_{t_{0}}^{\mathbb{T}} I_{t}^{\alpha} y\left(t_{0}\right)=0$.

Theorem 3 (See Theorem 24 of [24]). If $f: \mathcal{J} \times \mathbb{R} \rightarrow \mathbb{R}$ is a rd-continuous bounded function for which there exists $M>0$ such that $|f(t, y)| \leq M$ for all $t \in \mathcal{J}$ and $y \in \mathbb{R}$, then problem (1) has a solution on $\mathcal{J}$.

\section{Main Results}

We begin by proving formulas of integration by parts in Section 3.1, which are then used in Section 3.2 to prove necessary optimality conditions for nonlinear Riemann-Liouville fractional optimal control problems (FOCPs) on time scales. 


\subsection{Fractional integration by parts on time scales}

Our first result gives integration by parts formulas for fractional integrals and derivatives on time scales. For the relation between integration on time scales and Lebesgue integration we refer the reader to [29].

Theorem 4 Let $\alpha>0, p, q \geq 1$, and $\frac{1}{p}+\frac{1}{q} \leq 1+\alpha$, where $p \neq 1$ and $q \neq 1$ in the case when $\frac{1}{p}+\frac{1}{q}=1+\alpha$. Moreover, let

$$
{ }_{a}^{\mathbb{T}} I_{t}^{\alpha}\left(L_{p}\right):=\left\{f: f={ }_{a}^{\mathbb{T}} I_{t}^{\alpha} g, g \in L_{p}(a, b)\right\}
$$

and

$$
{ }_{t}^{\mathbb{T}} I_{b}^{\alpha}\left(L_{p}\right):=\left\{f: f={ }_{t}^{\mathbb{T}} I_{b}^{\alpha} g, g \in L_{p}(a, b)\right\} .
$$

The following integration by parts formulas hold.

(a) If $\varphi \in L_{p}(a, b)$ and $\psi \in L_{q}(a, b)$, then

$$
\int_{a}^{b} \varphi(t)\left({ }_{a}^{\mathbb{T}} I_{t}^{\alpha} \psi\right)(t) \Delta t=\int_{a}^{b} \psi(t)\left({ }_{t}^{\mathbb{T}} I_{b}^{\alpha} \varphi\right)(t) \Delta t
$$

(b) If $g \in{ }_{t}^{\mathbb{T}} I_{b}^{\alpha}\left(L_{p}\right)$ and $f \in{ }_{a}^{\mathbb{T}} I_{t}^{\alpha}\left(L_{q}\right)$, then

$$
\int_{a}^{b} g(t)\left({ }_{a}^{\mathbb{T}} D_{t}^{\alpha} f\right)(t) \Delta t=\int_{a}^{b} f(t)\left({ }_{t}^{\mathbb{T}} D_{b}^{\alpha} g\right)(t) \Delta t
$$

(c) For Caputo fractional derivatives, if $g \in{ }_{t}^{\mathbb{T}} I_{b}^{\alpha}\left(L_{p}\right)$ and $f \in{ }_{a}^{\mathbb{T}} I_{t}^{\alpha}\left(L_{q}\right)$, then

$$
\int_{a}^{b} g(t)\left({ }_{a}^{\mathbb{T} C} D_{t}^{\alpha} f\right)(t) \Delta t=\left[{ }_{t}^{\mathbb{T}} I_{b}^{1-\alpha} g(t) \cdot f(t)\right]_{a}^{b}+\int_{a}^{b} f(\sigma(t))\left({ }_{t}^{\mathbb{T}} D_{b}^{\alpha} g\right)(t) \Delta t
$$

and

$$
\int_{a}^{b} g(t)\left({ }_{t}^{\mathbb{T} C} D_{b}^{\alpha} f\right)(t) \Delta t=-\left[{ }_{a}^{\mathbb{T}} I_{t}^{1-\alpha} g(t) \cdot f(t)\right]_{a}^{b}+\int_{a}^{b} f(\sigma(t))\left({ }_{a}^{\mathbb{T}} D_{t}^{\alpha} g\right)(t) \Delta t .
$$

Proof (a) If $\varphi \in L_{p}(a, b)$ and $\psi \in L_{q}(a, b)$, then, from Definition 7 , we get

$$
\int_{a}^{b} \varphi(t)\left({ }_{a}^{\mathbb{T}} I_{t}^{\alpha} \psi\right)(t) \Delta t=\int_{a}^{b} \varphi(t)\left(\int_{a}^{t} \frac{(t-s)^{\alpha-1}}{\Gamma(\alpha)} \psi(s) \Delta s\right) \Delta t
$$

Interchanging the order of integrals (see [24]), we reach at

$$
\int_{a}^{b} \varphi(t)\left({ }_{a}^{\mathbb{T}} I_{t}^{\alpha} \psi\right)(t) \Delta t=\int_{a}^{b} \psi(t)\left({ }_{t}^{\mathbb{T}} I_{b}^{\alpha} \varphi\right)(t) \Delta t .
$$

(b) If $g \in{ }_{t}^{\mathbb{T}} I_{b}^{\alpha}\left(L_{p}\right)$ and $f \in{ }_{a}^{\mathbb{T}} I_{t}^{\alpha}\left(L_{q}\right)$, then, from Definition 8 , we get 


$$
\int_{a}^{b} g(t)\left({ }_{a}^{\mathbb{T}} D_{t}^{\alpha} f\right)(t) \Delta t=\int_{a}^{b} g(t)\left(\frac{1}{\Gamma(1-\alpha)}\left(\int_{a}^{t}(t-s)^{-\alpha} f(s) \Delta s\right)^{\Delta}\right) \Delta t .
$$

Interchanging the order of integrals, we obtain that

$$
\int_{a}^{b} g(t)\left({ }_{a}^{\mathbb{T}} D_{t}^{\alpha} f\right)(t) \Delta t=\int_{a}^{b} f(t)\left({ }_{t}^{\mathbb{T}} D_{b}^{\alpha} g\right)(t) \Delta t .
$$

(c) If $g \in{ }_{t}^{\mathbb{T}} I_{b}^{\alpha}\left(L_{p}\right)$ and $f \in{ }_{a}^{\mathbb{T}} I_{t}^{\alpha}\left(L_{q}\right)$, then, from Definition 9, we get

$$
\int_{a}^{b} g(t)\left({ }_{a}^{\mathbb{T} C} D_{t}^{\alpha} f\right)(t) \Delta t=\int_{a}^{b} g(t)\left(\frac{1}{\Gamma(1-\alpha)} \int_{a}^{t}(t-s)^{-\alpha} f^{\Delta}(s) \Delta s\right) \Delta t .
$$

Interchanging the order of the integrals, and by using integration by parts on time scales, we conclude that

$$
\int_{a}^{b} g(t)\left({ }_{a}^{\mathbb{T} C} D_{t}^{\alpha} f\right)(t) \Delta t=\int_{a}^{b} f(\sigma(t))\left({ }_{t}^{\mathbb{T}} D_{b}^{\alpha} g\right)(t) \Delta t+\left[{ }_{t}^{\mathbb{T}} I_{b}^{1-\alpha} g(t) \cdot f(t)\right]_{a}^{b} .
$$

The second relation is obtained in a similar way.

\subsection{Nonlinear Riemann-Liouville FOCPs on time scales}

Let $\mathbb{T}$ be a given time scale with $t_{0}, t_{f} \in \mathbb{T}$ and let us consider a control system given by the fractional differential equation

$$
{ }_{t_{0}}^{\mathbb{T}} D_{t}^{\alpha} x(t)=f(x(t), u(t), t), \quad t \in \mathbb{T},
$$

subject to

$$
{ }_{t_{0}}^{\mathbb{T}} I_{t}^{1-\alpha} x\left(t_{0}\right)=x_{0}
$$

where $x(t) \in \mathbb{R}^{n}$ and $u(t) \in \mathbb{R}^{m}$ are the state and control vectors, respectively, function $f: \mathbb{R}^{n \times m \times 1} \rightarrow \mathbb{R}^{n}$ is a nonlinear vector function, and $x_{0} \in \mathbb{R}^{n}$ is the specified initial state vector. A similar problem is studied in [14] for problems involving $\mathrm{AB}$ derivatives in Caputo sense on continuous time scales. Here we study it within Riemann-Liouville derivatives on arbitrary time scales. In order to achieve a desired behavior in terms of performance requirements, we select a cost index for the dynamical system (5)-(6). In selecting the performance index, the designer attempts to define a mathematical expression that, when minimized, indicates that the system is performing in the most desirable manner. Thus, choosing a performance cost index is a translation of system's physical requirements into mathematical terms [13]. For the fractional dynamic system (5)-(6), we choose the following performance index:

$$
J[x, u]=\int_{t_{0}}^{t_{f}} L(x(t), u(t), t) \Delta t \longrightarrow \min ,
$$


where $L: \mathbb{R}^{n \times m \times 1} \rightarrow \mathbb{R}$ is a scalar function. In the following, we derive a necessary optimality condition corresponding to the considered fractional optimal control problem (5)-(7). Under given considerations, the following theorem holds true.

Theorem 5 (Necessary optimality conditions) Let $(x(\cdot), u(\cdot))$ be a minimizer of problem (5)-(7). Then, there exists a function $\lambda(\cdot)$ for which the triplet $(x(\cdot), \lambda(\cdot), u(\cdot))$ satisfies:

(i) the Hamiltonian system

$$
\begin{cases}{ }_{t_{0}} D_{t}^{\alpha} x(t)=\frac{\partial \mathcal{H}}{\partial \lambda}(x(t), \lambda(t), u(t), t), & t \in \mathbb{T}, \\ { }_{t}^{\mathbb{T}} D_{t_{f}}^{\alpha} \lambda(t)=\frac{\partial \mathcal{H}}{\partial x}(x(t), \lambda(t), u(t), t), & t \in \mathbb{T}\end{cases}
$$

(ii) the stationary condition

$$
\frac{\partial \mathcal{H}}{\partial u}(x(t), \lambda(t), u(t), t)=0, \quad t \in \mathbb{T},
$$

where $\mathcal{H}$ is a scalar function, called the Hamiltonian, defined by

$$
\mathcal{H}(x, \lambda, u, t)=L(x, u, t)+\lambda^{T} f(x, u, t) .
$$

Proof To deduce the necessary optimality conditions that the optimal pair $(x(\cdot), u(\cdot))$ must satisfy, we use the Lagrange multiplier technique to adjoin the dynamic constraint (5) to the performance index (7). Thus, we form the augmented functional

$$
J_{a}[x, \lambda, u]=\int_{t_{0}}^{t_{f}}\left[\mathcal{H}(x(t), \lambda(t), u(t), t)-\lambda^{T}(t)_{t_{0}}^{\mathbb{T}} D_{t}^{\alpha} x(t)\right] \Delta t,
$$

where $\lambda(t) \in \mathbb{R}^{n}$ is the Lagrange multiplier, also known as the costate or adjoint variable. Taking the first variation of the augmented performance index $J_{a}[x, \lambda, u]$ given by (11), we obtain that

$$
\begin{aligned}
\delta J_{a}[x, \lambda, u]=\int_{t_{0}}^{t_{f}} & \left\{\left[\frac{\partial \mathcal{H}}{\partial x}\right]^{T} \delta x(t)+\left[\frac{\partial \mathcal{H}}{\partial \lambda}-{ }_{t_{0}}^{\mathbb{T}} D_{t}^{\alpha} x(t)\right]^{T} \delta \lambda(t)\right. \\
+ & {\left.\left[\frac{\partial \mathcal{H}}{\partial u}\right]^{T} \delta u(t)-\lambda^{T}(t)_{t_{0}}^{\mathbb{T}} D_{t}^{\alpha} \delta x(t)\right\} \Delta t . }
\end{aligned}
$$

Using the fractional integration by parts formula (4), the last integral in (12) can be written as

$$
\int_{t_{0}}^{t_{f}} \lambda^{T}(t){ }_{t_{0}}^{\mathbb{T}} D_{t}^{\alpha} \delta x(t) \Delta t=\int_{t_{0}}^{t_{f}}\left({ }_{t}^{\mathbb{T}} D_{t_{f}}^{\alpha} \lambda(t)\right)^{T} \delta x(t) \Delta t .
$$

Using (13) in (12), we deduce that 


$$
\begin{aligned}
\delta J_{a}[x, \lambda, u]=\int_{t_{0}}^{t_{f}} & \left\{\left[\frac{\partial \mathcal{H}}{\partial x}-{ }_{t}^{\mathbb{T}} D_{t_{f}}^{\alpha} \lambda(t)\right]^{T} \delta x(t)+\left[\frac{\partial \mathcal{H}}{\partial \lambda}-{ }_{t_{0}}^{\mathbb{T}} D_{t}^{\alpha} x(t)\right]^{T} \delta \lambda(t)\right. \\
+ & {\left.\left[\frac{\partial \mathcal{H}}{\partial u}\right]^{T} \delta u(t)\right\} \Delta t . }
\end{aligned}
$$

The necessary condition for an extremal asserts that the first variation of $J_{a}[x, \lambda, u]$ must vanish along the extremal for all independent variations $\delta x(t), \delta \lambda(t)$, and $\delta u(t)$. Because of this, all factors multiplying a variation in Eq. (14) must vanish. We obtain conditions (8)-(9).

Equations (8)-(9) represent the Euler-Lagrange equations of the FOCP (5)-(7). Note that Theorem 5 covers fractional optimal control problems defined on isolated time scales with a non-constant graininess, as well as variational problems on time scales that are partially continuous and partially discrete, i.e., on hybrid time scales.

\subsection{An illustrative example}

Let $\mathbb{T}$ be a time scale with $0, T \in \mathbb{T}$. Consider the control system

$$
{ }_{0}^{\mathbb{T}} D_{t}^{\alpha} x(t)=u(t), \quad t \in[0, T]_{\mathbb{T}},
$$

subject to the initial condition

$$
{ }_{0}^{\mathbb{T}} I_{t}^{1-\alpha} x(0)=x_{0},
$$

where the control $u$ belongs to $L^{2}$. Consider the problem of minimizing

$$
J[x, u]=\frac{1}{2}\left(\|x-z\|_{L^{2}}^{2}+N\|u\|_{L^{2}}^{2}\right)
$$

subject to (15)-(16), where $z \in L^{2}$ and $N>0$ are fixed/given. In agreement with Theorem 5, the optimal control $u$ is characterized by (15)-(16) with the adjoint system

$$
{ }_{t}^{\mathbb{T}} D_{T}^{\alpha} \lambda(t)=x(t)-z(t), \quad t \in[0, T]_{\mathbb{T}},
$$

and with the optimality condition

$$
u(t)=-\frac{\lambda(t)}{N}
$$




\section{Conclusion}

We studied optimal control problems for fractional initial values systems involving fractional-time derivatives on time scales. As a main result, a necessary optimality condition is proved. In the formulation of the optimal control problem, the control $u$ takes values in $\mathbb{R}^{m}$. As future work, it would be interesting to consider the case where the control takes values on a closed subset of $\mathbb{R}^{m}$. This question is far from being trivial [28, 35] and needs further developments.

Acknowledgements Torres has been partially supported by Fundação para a Ciência e a Tecnologia (FCT) through CIDMA, project UID/MAT/04106/2019. The authors are grateful to two anonymous referees for several pertinent questions and comments.

\section{References}

1. S. Abbas, M. Benchohra and G. M. N'Guérékata, Topics in fractional differential equations, Developments in Mathematics, 27, Springer, New York, 2012.

2. T. Abdeljawad, R. Mert and D. F. M. Torres, Variable order Mittag-Leffler fractional operators on isolated time scales and application to the calculus of variations. In Fractional Derivatives with Mittag-Leffler Kernel, J. F. Gómez et al. (Eds.), Springer Nature Switzerland AG 2019, Studies in Systems, Decision and Control 194, 35-47. arXiv: 1809.02029

3. T. Abdeljawad and D. F. M. Torres, Symmetric duality for left and right RiemannLiouville and Caputo fractional differences, Arab J. Math. Sci. 23 (2017), no. 2, 157-172. arXiv: 1607.03458

4. R. P. Agarwal and M. Bohner, Basic calculus on time scales and some of its applications, Results Math. 35 (1999), no. 1-2, 3-22.

5. R. P. Agarwal, M. Bohner, D. O'Regan and A. Peterson, Dynamic equations on time scales: a survey, J. Comput. Appl. Math. 141 (2002), no. 1-2, 1-26.

6. O. P. Agrawal, Formulation of Euler-Lagrange equations for fractional variational problems, J. Math. Anal. Appl. 272 (2002), no. 1, 368-379.

7. O. P. Agrawal, A general formulation and solution scheme for fractional optimal control problems, Nonlinear Dynam. 38 (2004), no. 1-4, 323-337.

8. A. Ahmadkhanlu and M. Jahanshahi, On the existence and uniqueness of solution of initial value problem for fractional order differential equations on time scales, Bull. Iranian Math. Soc. 38 (2012), no. 1, 241-252.

9. R. Almeida, D. Tavares and D. F. M. Torres, The variable-order fractional calculus of variations, SpringerBriefs in Applied Sciences and Technology, Springer, Cham, 2019. arXiv: 1805.00720

10. B. Aulbach and S. Hilger, A unified approach to continuous and discrete dynamics, in Qualitative theory of differential equations (Szeged, 1988), 37-56, Colloq. Math. Soc. János Bolyai, 53, North-Holland, Amsterdam, 1990.

11. G. M. Bahaa and Q. Tang, Optimality conditions of fractional diffusion equations with weak Caputo derivatives and variational formulation, J. Fract. Calc. Appl. 9 (2018), no. 1, 100-119.

12. E. G. Bajlekova, Fractional evolution equations in Banach spaces, Eindhoven University of Technology, Eindhoven, 2001.

13. D. Baleanu, A. Jajarmi and M. Hajipour, A new formulation of the fractional optimal control problems involving Mittag-Leffler nonsingular kernel, J. Optim. Theory Appl. 175 (2017), no. $3,718-737$. 
14. D. Baleanu and S. I. Muslih, Lagrangian formulation of classical fields within RiemannLiouville fractional derivatives, Phys. Scripta 72 (2005), no. 2-3, 119-121.

15. N. R. O. Bastos, Fractional calculus on time scales, $\mathrm{PhD}$ thesis (under supervision of D. F. M. Torres), University of Aveiro, 2012. arXiv: 1202.2960

16. N. R. O. Bastos, R. A. C. Ferreira and D. F. M. Torres, Necessary optimality conditions for fractional difference problems of the calculus of variations, Discrete Contin. Dyn. Syst. 29 (2011), no. 2, 417-437. arXiv: 1007.0594

17. N. R. O. Bastos, R. A. C. Ferreira and D. F. M. Torres, Discrete-time fractional variational problems, Signal Process. 91 (2011), no. 3, 513-524. arXiv: 1005.0252

18. N. R. O. Bastos, D. Mozyrska and D. F. M. Torres, Fractional derivatives and integrals on time scales via the inverse generalized Laplace transform, Int. J. Math. Comput. 11 (2011), J11, 1-9. arXiv: 1012.1555

19. B. Bayour, A. Hammoudi and D. F. M. Torres, A truly conformable calculus on time scales, Glob. Stoch. Anal. 5 (2018), no. 1, 1-14. arXiv: 1705.08928

20. B. Bayour and D. F. M. Torres, Complex-valued fractional derivatives on time scales, in Differential and difference equations with applications, 7 9-87, Springer Proc. Math. Stat., 164, Springer, 2016. arXiv: 1511.02153

21. B. Bayour and D. F. M. Torres, Structural derivatives on time scales, Commun. Fac. Sci. Univ. Ank. Ser. A1 Math. Stat. 68 (2019), no. 1, 1186-1196. arXiv: 1811.09474

22. N. Benkhettou, A. M. C. Brito da Cruz and D. F. M. Torres, A fractional calculus on arbitrary time scales: fractional differentiation and fractional integration, Signal Process. 107 (2015), 230-237. arXiv: 1405.2813

23. N. Benkhettou, A. M. C. Brito da Cruz and D. F. M. Torres, Nonsymmetric and symmetric fractional calculi on arbitrary nonempty closed sets, Math. Methods Appl. Sci. 39 (2016), no. 2, 261-279. arXiv: 1502.07277

24. N. Benkhettou, A. Hammoudi and D. F. M. Torres, Existence and uniqueness of solution for a fractional Riemann-Liouville initial value problem on time scales, J. King Saud Univ. Sci. 28 (2016), no. 1, 87-92. arXiv: 1508.00754

25. N. Benkhettou, S. Hassani and D. F. M. Torres, A conformable fractional calculus on arbitrary time scales, J. King Saud Univ. Sci. 28 (2016), no. 1, 93-98. arXiv:1505.03134

26. M. Bohner and A. Peterson, Dynamic equations on time scales, Birkhäuser Boston, Inc., Boston, MA, 2001.

27. M. Bohner and A. Peterson, Advances in dynamic equations on time scales, Birkhäuser Boston, Boston, MA, 2003.

28. L. Bourdin, O. Stanzhytskyi and E. Trélat, Addendum to Pontryagin's maximum principle for dynamic systems on time scales, J. Difference Equ. Appl. 23 (2017), no. 10, 1760-1763.

29. A. Cabada and D. R. Vivero, Expression of the Lebesgue $\Delta$-integral on time scales as a usual Lebesgue integral: application to the calculus of $\Delta$-antiderivatives, Math. Comput. Modelling 43 (2006), no. 1-2, 194-207.

30. M. C. Caputo and D. F. M. Torres, Duality for the left and right fractional derivatives, Signal Process. 107 (2015), 265-271. arXiv: 1409.5319

31. M. Dryl and D. F. M. Torres, Direct and inverse variational problems on time scales: a survey, in Modeling, dynamics, optimization and bioeconomics. II, 223-265, Springer Proc. Math. Stat., 195, Springer, Cham, 2017. arXiv:1601.05111

32. G. S. F. Frederico and D. F. M. Torres, A formulation of Noether's theorem for fractional problems of the calculus of variations, J. Math. Anal. Appl. 334 (2007), no. 2, 834-846. arXiv:math/0701187

33. G. S. F. Frederico and D. F. M. Torres, Fractional optimal control in the sense of Caputo and the fractional Noether's theorem, Int. Math. Forum 3 (2008), no. 9-12, 479-493. arXiv:0712.1844

34. E. Hernández, D. O'Regan and K. Balachandran, On recent developments in the theory of abstract differential equations with fractional derivatives, Nonlinear Anal. 73 (2010), no. 10, 3462-3471. 
35. D. Idczak and R. Kamocki, Existence of optimal solutions to Lagrange problem for a fractional nonlinear control system with Riemann-Liouville derivative, Math. Control Relat. Fields 7 (2017), no. 3, 449-464.

36. O. Lavrova, V. Mogylova, O. Stanzhytskyi and O. Misiats, Approximation of the optimal control problem on an interval with a family of optimization problems on time scales, Nonlinear Dyn. Syst. Theory 17 (2017), no. 3, 303-314.

37. A. B. Malinowska, T. Odzijewicz and D. F. M. Torres, Advanced methods in the fractional calculus of variations, SpringerBriefs in Applied Sciences and Technology, Springer, Cham, 2015.

38. K. Mekhalfi and D. F. M. Torres, Generalized fractional operators on time scales with application to dynamic equations, Eur. Phys. J. Special Topics 226 (2017), no. 16-18, 3489-3499. arXiv: 1804.02536

39. G. M. Mophou, Optimal control of fractional diffusion equation, Comput. Math. Appl. 61 (2011), no. 1, 68-78.

40. G. Mophou and G. M. N'Guérékata, Optimal control of a fractional diffusion equation with state constraints, Comput. Math. Appl. 62 (2011), no. 3, 1413-1426.

41. D. Mozyrska, D. F. M. Torres and M. Wyrwas, Solutions of systems with the Caputo-Fabrizio fractional delta derivative on time scales, Nonlinear Anal. Hybrid Syst. 32 (2019), 168-176. arXiv: 1812.00266

42. E. R. Nwaeze and D. F. M. Torres, Chain rules and inequalities for the BHT fractional calculus on arbitrary timescales, Arab. J. Math. (Springer) 6 (2017), no. 1, 13-20. arXiv: 1611.09049

43. N. Özdemir, D. Karadeniz and B. B. İskender, Fractional optimal control problem of a distributed system in cylindrical coordinates, Phys. Lett. A 373 (2009), no. 2, 221-226.

44. I. Podlubny, Fractional differential equations, Mathematics in Science and Engineering, 198, Academic Press, San Diego, CA, 1999.

45. M. R. Sidi Ammi and D. F. M. Torres, Existence and uniqueness results for a fractional Riemann-Liouville nonlocal thermistor problem on arbitrary time scales, J. King Saud Univ. Sci. 30 (2018), no. 3, 381-385. arXiv: 1703.05439

46. M. R. Sidi Ammi and D. F. M. Torres, Analysis of fractional integro-differential equations of thermistor type. In Handbook of Fractional Calculus with Applications, Vol 1: Basic Theory, A. Kochubei, Y. Luchko (Eds.), De Gruyter, Berlin, Boston, 2019, 327-346. arXiv: 1807.01529 